\title{
Expériences économiques en économie agricole. État des lieux et dynamiques de recherche
}

Experimental Economics Applied to Agricultural Issues: Taking Stock and Research Perspectives

Douadia Bougherara, Marielle Brunette, Christoph Heinzel, Lisette Ibanez, Laurent Muller et Sabrina Teyssier

\section{(2) OpenEdition}

\section{Journals}

Édition électronique

URL : http://journals.openedition.org/economierurale/5341

DOI : 10.4000/economierurale.5341

ISSN : 2105-2581

Éditeur

Société Française d'Économie Rurale (SFER)

Édition imprimée

Date de publication : 15 décembre 2017

Pagination : $29-48$

ISSN : 0013-0559

Référence électronique

Douadia Bougherara, Marielle Brunette, Christoph Heinzel, Lisette Ibanez, Laurent Muller et Sabrina Teyssier, «Expériences économiques en économie agricole. État des lieux et dynamiques de recherche », Économie rurale [En ligne], 362 | Novembre-décembre 2017, mis en ligne le 15 décembre 2019, consulté le 05 janvier 2020. URL : http://journals.openedition.org/economierurale/5341 ; DOI : 10.4000/economierurale.5341 


\title{
Expériences économiques en économie agricole État des lieux et dynamiques de recherche
}

\author{
Douadia BOUGHERARA • LAMETA, INRA, Université Montpellier, CNRS, SupAgro, UPVM3, \\ Montpellier, France \\ douadia.bougherara@inra.fr \\ Marielle BRUNETTE • LEF, INRA, Agroparistech, Nancy, France \\ marielle.brunette@inra.fr \\ Christoph HEINZEL • INRA, SMART-LERECO, INRA, AGROCAMPUS, Rennes, France \\ christoph.heinzel@inra.fr \\ Lisette IBANEZ • LAMETA, INRA, Université Montpellier, CNRS, SupAgro, UPVM3, Montpellier, \\ France \\ lisette.ibanez@inra.fr \\ Laurent MULLER • GAEL, UMR 1215, INRA, CNRS, Grenoble INP, Université Grenoble-Alpes, \\ Grenoble \\ laurent.muller@inra.fr
}

Sabrina TEYSSIER • GAEL, INRA, CNRS, Grenoble INP, Université Grenoble-Alpes, Grenoble.

sabrina.teyssier@inra.fr

L'économie expérimentale est de plus en plus utilisée en économie agricole. Cet article rend compte d'une École Chercheurs organisée en 2015 par le Département SAE2 (Sciences Sociales, Agriculture et Alimentation, Espace et Environnement) de I'INRA afin de faire un état des lieux des travaux employant cette méthode et de discuter de son apport à l'économie agricole. Les auteurs examinent les défis méthodologiques comme les incitations monétaires et le biais hypothétique, les validités interne et externe ainsi que l'utilisation de la tromperie en lien avec les spécificités de la recherche en économie agricole, puis la portée académique et sociétale de travaux expérimentaux. Encore jeune dans ce champ, l'économie expérimentale ouvre la voie à des questions de recherche prometteuses.

MOTS-CLÉS : économie expérimentale, économie agricole, biais hypothétique, validité interne et externe, tromperie

\section{Experimental Economics Applied to Agricultural Issues: Taking Stock and Research Perspectives}

Experimental economics is increasingly used in the field of agricultural economics. This article reports the main insights of a summer school organized in 2015 by the INRA SAE2 Department (Social Sciences, Agriculture and Food, Rural Development and Environment) in order to review existing research using experiments and to discuss the relevance of experimental methods in this area. We discuss the main methodological issues like financial incentives and the hypothetical bias, internal and external validity, and deception. We also show the relevance of economic experiments in helping public decision-makers and firms. We argue that economic experiments are a promising method in this field. (JEL: C9, Q1, Q5, Q18)

KEYWORDS: design of experiments, agriculture, environmental economics, food policy 
'économie expérimentale est de plus en -plus utilisée en économie agricole ${ }^{1}$. Du fait de la nature finalisée des recherches qui visent à la fois l'aide à la décision publique et privée, l'application de la méthode expérimentale aux questions de l'économie agricole fait face à des verrous méthodologiques. L'objectif de cet article est d'apporter une meilleure vision de la recherche en économie agricole qui utilise la méthodologie expérimentale et de discuter les questions méthodologiques sous-jacentes. Cet article fait suite à l'organisation d'une École Chercheurs par le Département SAE2 (Sciences Sociales, Agriculture et Alimentation, Espace et Environnement) de l'INRA (Institut National de la Recherche Agronomique) intitulée Économie expérimentale: état des lieux et dynamiques de recherche à l'INRA qui s'est déroulée à Montpellier du 30 septembre au 2 octobre 2015.

Une expérience en économie consiste à reconstituer une situation économique simplifiée dans un environnement contrôlé. L'expérimentateur choisit les variables d'intérêt ainsi que les autres variables, explicatives ou de contrôle, dont il maîtrise les valeurs afin de déterminer l'impact ceteris paribus de celles-ci sur les comportements individuels ou collectifs des participants. Un tel contrôle permet l'identification de l'effet causal de ces variables.

Pendant longtemps, l'économie a été qualifiée de science non expérimentale pour laquelle l'observation de données qui se révèlent de manière naturelle était

1. Nous utilisons le terme économie agricole de manière large. Il englobe l'analyse économique des questions relatives à l' agriculture, l'environnement et l'alimentation. Une analyse bibliométrique des publications en économie expérimentale basée sur plus de 7000 références bibliographiques met en évidence qu'au niveau international l'économie expérimentale est utilisée majoritairement pour aborder les questions d'alimentation, ensuite pour traiter d'environnement et enfin d'agriculture (Frappier, 2015). la seule possibilité. Dans leur manuel classique d'économie, Samuelson et Nordhaus (1985) expriment cette idée ${ }^{2}$ : «Le monde économique est extrêmement complexe. Il $\mathrm{y}$ a des millions de personnes et de firmes, des milliers de prix et d'industries. Un des moyens de déterminer la façon dont est régie l'économie est de conduire des expériences contrôlées. Une expérience contrôlée consiste à faire varier la variable étudiée lorsque toutes les autres variables sont maintenues constantes. Ainsi, un chercheur qui étudie l'impact de la saccharine sur le cancer chez les rats maintiendra toutes choses égales par ailleurs et fera varier uniquement la quantité de saccharine. Même air, même lumière, même type de rat. Les économistes n'ont pas un tel luxe pour tester les lois de l'économie. Ils ne peuvent pas conduire les expériences contrôlées que conduisent les chimistes ou les biologistes parce qu'ils ne peuvent pas facilement contrôler des facteurs importants autres que la variable étudiée. Comme les astronomes ou les météorologues, ils doivent en général se contenter largement de l'observation. »

Pourtant, en 2002, des travaux d'économie expérimentale ont été couronnés par l'attribution jointe du prix Nobel d'économie à l'économiste Vernon $\mathrm{Smith}^{3}$ pour avoir fait de l'expérience en laboratoire un instrument d'analyse économique empirique, en particulier dans l'étude de différentes structures de marché et au psychologue et économiste Daniel Kahneman pour avoir introduit en sciences économiques des acquis de la recherche en psychologie, en particulier concernant les jugements et les décisions en incertitude. L'économie est donc aussi une science

2. Traduction des auteurs.

3. Voir le site Internet de la Royal Swedish Academy of Sciences: http://www.kva.se/en// Prizes/Prize-winner-page/laureateId=551; http:// www.kva.se/en/Prizes/Prize-winner-page/ ?laureate $\mathrm{Id}=550$. 
expérimentale et de nombreux travaux s'appuient sur cette méthodologie ${ }^{4}$.

Les expériences remplissent un ou plusieurs des trois objectifs définis par Roth (1988) : (i) tester la théorie afin d'identifier des mécanismes non prédits par les modèles existants ; (ii) produire des faits afin d'identifier des régularités comportementales jusqu'alors non connues et susceptibles d'être intégrées dans les modèles théoriques; (iii) aider à la décision en testant les implications de la mise en place d'un nouveau mécanisme. Les deux premiers objectifs sont interdépendants et s'entretiennent mutuellement. Quant au troisième objectif, il s'agit d'évaluer les conséquences économiques d'un changement organisationnel ou institutionnel. Il est ainsi possible d'évaluer l'impact de la mise en place de politiques publiques diverses, différentes formes de régulation du marché ou encore différents systèmes organisationnels au sein de l'entreprise.

L'interaction entre avancées théoriques et empiriques est cruciale. Amorcée notamment par le développement des outils informatiques et statistiques, nous notons ces dernières années une croissance de la fréquence des tests empiriques pour valider des avancées théoriques. Les tests empiriques occupent en effet la majeure partie des publications dans les meilleures revues en sciences économiques depuis les vingt dernières années (Hamermesh, 2013; Treich, 2015). Dans ce contexte, un poids croissant est accordé aux données créées ex nihilo pour répondre à un questionnement spécifique, impliquant la réalisation

\footnotetext{
4. Nous pouvons également citer les travaux d'Elinor Ostrom et Alvin Roth (Ostrom, 2009 ; Roth, 2012) qui ont reçu le prix Nobel d'économie en 2009 et 2012, respectivement; voir le site Internet de la Royal Swedish Academy of Sciences : http://www.kva.se/en/Prizes/Prizewinner-page/ laureateId=718 ; http://www.kva.se/ en/Prizes/Prize-winner-page/ ?laureateId=792.
} d'expériences, soit sur le terrain, soit en laboratoire.

En économie agricole, nous trouvons principalement des expériences relevant de trois des quatre types définis par Harrison et List (2004) : (i) expériences en laboratoire (conventional laboratory experiments) : les participants sont en général des étudiants sélectionnés aléatoirement, le cadre est décontextualisé et les règles sont imposées par l'expérimentateur ; (ii) expériences en laboratoire avec sujets non standards (artefactual field experiments) : elles ressemblent aux expériences de laboratoire sauf que les participants ne sont pas des étudiants mais la population cible de l'étude (agriculteurs, consommateurs, dirigeants d'entreprises, etc.) ; (iii) expériences de terrain contrôlées (framed field experiments): elles ressemblent au type précédent mais avec un cadre contextualisé impliquant le produit évalué ou échangé dans l'expérience, la décision à réaliser ou l'information donnée aux sujets ; (iv) expériences naturelles (natural framed field experiments): les participants réalisent une tâche naturellement, sans savoir qu'ils font partie d'une expérience. Ce dernier type d'expérience est plus rare car le terrain d'observation doit pouvoir garantir le contrôle des variables explicatives.

Dans cet article, nous examinons les défis méthodologiques comme les incitations monétaires et le biais hypothétique, les validités interne et externe et l'utilisation de la tromperie, en lien avec les spécificités de la recherche en économie agricole, et la portée académique et sociétale de travaux expérimentaux.

\section{Enjeux méthodologiques de l'économie expérimentale en économie agricole}

Les présentations lors de l'École Chercheurs ont été regroupées selon les trois thématiques principales de recherche 
de l'INRA : l'agriculture, l'environnement et l'alimentation. En matière d'agriculture, il s'agit de s'intéresser à la production et aux marchés agricoles avec notamment l'étude du fonctionnement des exploitations, de l'organisation des échanges et des négociations internationales. Concernant l'environnement, la recherche se focalise sur la protection et la gestion des ressources naturelles avec des questionnements de développement durable. Enfin, pour l'alimentation, il s'agit d'étudier la consommation, la transformation et la distribution des produits alimentaires via, entres autres, les relations aliments-alimentation-santé, l'organisation et le fonctionnement des filières agroalimentaires.

En s'appuyant sur des expériences récentes, l'École Chercheurs a permis d'aborder les enjeux méthodologiques de l'économie expérimentale quand celle-ci est appliquée aux problématiques de l'économie agricole. Nous mettons l'accent sur trois principaux défis méthodologiques: (i) incitations monétaires et biais hypothétique ; (ii) validité interne et externe ; (iii) usage de la tromperie. Les défis ont été abordés lors de présentations d'articles de recherche ainsi que dans une série d'ateliers. Sur chacun des trois thèmes, agriculture, environnement et alimentation, deux travaux de recherche ont été présentés et sont résumés dans le tableau 1. Le choix des travaux s'est fait de manière à respecter une certaine hétérogénéité, à la fois en termes de questions de recherche (colonne 1), de types de jeux expérimentaux (colonne 2) et de populations (colonne 3 ). Les colonnes 4 et 5 complètent le tableau en présentant respectivement les résultats obtenus et les points de discussion relevés au cours de l'École Chercheurs.

$\mathrm{Au}$ sein de chaque thème (agriculture, environnement et alimentation), un questionnement principal, qui est fondamental dans la thématique, est soulevé et abordé selon deux perspectives distinctes, chaque perspective étant illustrée par un article de recherche. Pour le thème agriculture, les deux études portent sur l'analyse de la gestion du risque pour des producteurs (agriculteurs et pêcheurs). Une étude vise à révéler les préférences des agriculteurs vis-à-vis du risque (Reynaud et Couture, 2012), l'autre à évaluer l'efficacité de contrats de pêche avec pour objectif de limiter le risque d'épuisement d'une ressource commune (Mantilla et al., 2015). Pour le thème environnement, les deux études analysent l'impact de la mise en place de mécanismes ayant pour objectif la protection de l'environnement. Une étude teste la mise en place d'une politique de taxation (Rozan et al., 2015) et l'autre teste une politique d'information (Dubois et al., 2015). Pour le thème alimentation, les deux études mesurent l'impact d'un étiquetage environnemental sur les décisions d'achat des consommateurs. Une étude porte sur l'achat d'un bien spécifique qui est le vin (Bazoche et al., 2015), l'autre prend en compte l'achat d'un panier alimentaire composé de plusieurs produits (Lacroix et al., 2015).

Par ailleurs, l'ensemble des six études présentées permettent de nuancer deux types de protocoles et aussi deux types de sujets. Une différence importante entre les protocoles repose sur l'interconnexion entre les participants à l'expérience. Lorsqu'il s'agit de révéler les préférences des individus, comme des préférences visà-vis du risque ou pour des biens privés, le protocole est généralement fondé sur des décisions individuelles et donc construit afin que celles-ci n'engagent que les sujets en conduisant à des conséquences uniquement sur leur propre rémunération. À l'inverse, pour une question de recherche portant sur la coordination entre individus, pour la protection d'une ressource commune par exemple, il est important que les participants à l'expérience interagissent et donc que leurs décisions impactent à la 
RECHERCHE

Douadia BOUGHERARA, Marielle BRUNETTE, Christoph HEINZEL, Lisette IBANEZ,

Laurent MULLER, Sabrina TEYSSIER

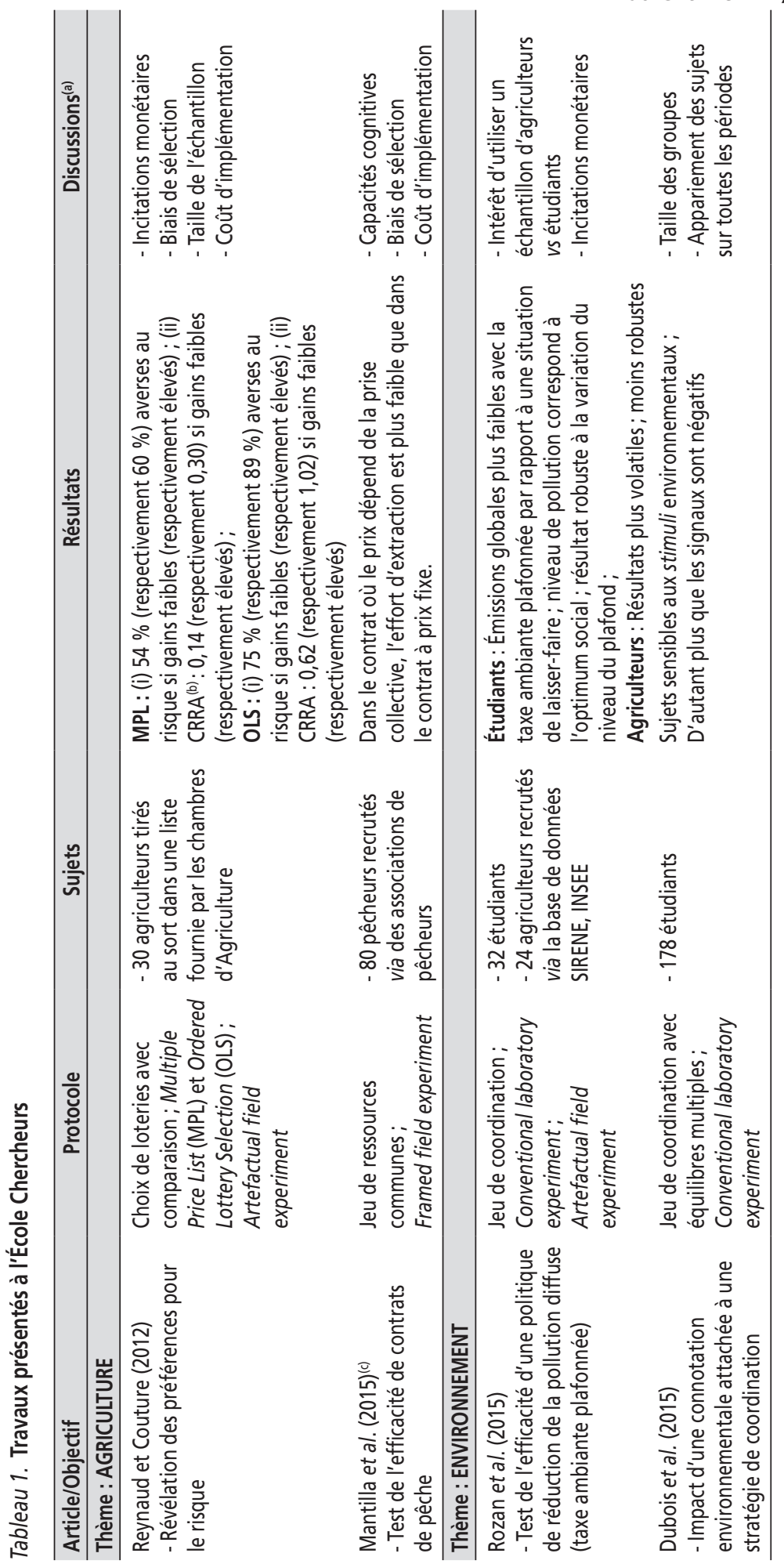




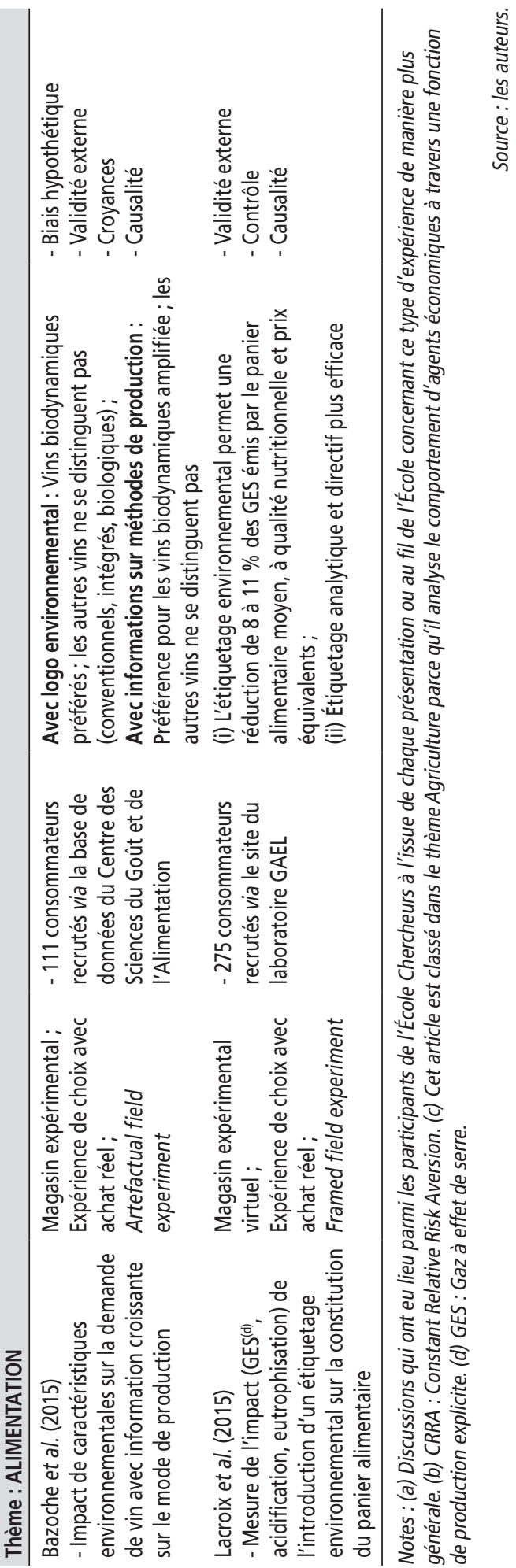


fois leur propre rémunération mais également la rémunération d'autres participants. Trois des études présentées reposent sur des décisions individuelles (Reynaud et Couture, 2012; Bazoche et al., 2015; Lacroix et al., 2015) et les trois autres sur des décisions avec interactions entre individus (Mantilla et al., 2015; Rozan et al., 2015 ; Dubois et al., 2015). Le type de sujets participant à l'expérience est également central et dépend de la question de recherche posée. Les thématiques de recherche en économie agricole étant principalement finalisées requièrent le plus souvent de faire appel à des individus représentatifs d'une catégorie particulière telle que des agriculteurs, des pêcheurs ou des consommateurs. Pour des questions plus généralistes en économie agricole, il peut également être nécessaire de faire appel à des étudiants comme dans Rozan et al. (2015) et Dubois et al. (2015).

\section{Incitations monétaires et biais hypothétique}

Les incitations monétaires démarquent généralement les études économiques de celles en psychologie (Kagel et Roth, 1995). La rémunération des sujets est pour la majorité des économistes nécessaire en raison de ses vertus incitatives. Pour Plott (1982), l'expérimentation en laboratoire et de terrain est utile en économie car elle correspond à un processus réel de décision avec de vraies personnes qui réalisent des tâches définies et dont les décisions et les efforts pour ces tâches ont des conséquences réelles. En effet, la rémunération affecte à la fois le niveau d'effort fourni par le participant et son état d'esprit lorsqu'il aborde l'expérience (Etchart-Vincent, 2006). Ce que l'expérimentaliste cherche à éviter, c'est notamment le biais hypothétique. La nécessité d'inciter financièrement les sujets fait cependant l'objet de discussions dans la littérature. Nous y revenons ci-dessous. Nous présentons tout d'abord le biais hypothétique que les économistes cherchent à minimiser en mettant en place des incitations monétaires.

Le biais hypothétique est maintenant bien documenté (voir par exemple Loomis, 2011) notamment dans le cadre de méthodes d'évaluation comme l'évaluation contingente, pour lesquelles aucune incitation monétaire n'est mise en place. Le biais hypothétique est défini comme l'écart entre la réponse fournie par un participant dans une expérience hypothétique et ce qu'il aurait indiqué en situation réelle ou incitée. Par exemple, Holt et Laury (2002) montrent que le choix d'une loterie dépend de sa réalisation. Ils montrent notamment que les individus affichent une plus grande aversion pour le risque lorsque les loteries aboutissent à de véritables gains monétaires. Les causes sous-jacentes du biais hypothétique ne sont pas encore suffisamment comprises et son explication représente l'un des questionnements majeurs de l'analyse économique des préférences (Mitani et Flores, 2014). Les raisons de l'existence du biais hypothétique sont multiples. L'une d'entre elles réside dans la désirabilité sociale. En effet, pour certaines questions faisant appel aux préférences sociales des participants, ceuxci sont tentés de répondre de la manière la plus acceptable socialement alors que leur choix pourrait être différent s'ils recevaient une compensation monétaire dépendante de leurs décisions ${ }^{5}$. Les participants peuvent également ne pas révéler leurs vraies préférences dans une expérience hypothétique, notamment parce qu'ils ont

5. Ce point est à nuancer. En effet, de nombreux travaux expérimentaux ont montré que même en présence d'incitations monétaires, les individus se soucient des comportements des autres et peuvent exprimer le désir de suivre une norme (voir par exemple, Camerer et Fehr [2004]). Dans ce cas, les comportements observés révèlent que certains individus ont des préférences sociales malgré le coût monétaire que cela peut impliquer. Il ne s'agit pas ici d'un biais mais de l'expression réelle des préférences sociales des individus. 
du mal à se projeter dans la tâche proposée par l'expérimentateur ou parce qu'ils essaient de répondre de manière stratégique en pensant que leurs réponses auront des incidences réelles, par exemple concernant des questions relatives à la tarification d'un produit ou service, à la mise en place d'une politique publique, ou encore à la mise en marché d'un nouveau bien (Lusk et al., 2007).

Cinq des six travaux présentés à l'École Chercheurs utilisent des incitations monétaires (Bazoche et al. (2015); Dubois et al. (2015) ; Lacroix et al. (2015); Mantilla et al. (2015) ; Rozan et al. (2015)). Il existe cependant des cas où il est plus difficile de les mettre en place. Dans le domaine de l'agriculture, les expérimentateurs font souvent appel à des agriculteurs pour participer aux expériences plutôt qu'à des étudiants ${ }^{6}$. Les agriculteurs, ayant des caractéristiques spécifiques et un métier associé à des problématiques propres, ont des motivations différentes des étudiants. Par exemple, l'agriculture étant un secteur où l'intervention publique est élevée, les agriculteurs ont peut-être plus confiance dans leur capacité à influencer les choix politiques par leurs réponses à l'expérience. Les motivations des agriculteurs dans l'expérience n'étant pas que financières, la capacité des expériences à contrôler les incitations doit être nuancée. De plus, la rémunération à l'expérience doit au moins couvrir le coût d'opportunité des participants, plus élevé dans le cas d'agriculteurs que d'étudiants. C'est d'autant plus vrai que la recherche concerne des choix

\footnotetext{
6. La mobilisation d'étudiants comme sujets dans les expériences ne pose pas de problème lorsqu'il s'agit de tester une théorie qui ne dit rien sur les caractéristiques des agents. Mais, pour l'instant, les travaux en agriculture ont utilisé des agriculteurs parce que la question de recherche exigeait ce type d'échantillon. Par exemple, la mesure de préférences pour le risque chez les agriculteurs nécessite de mobiliser un échantillon d'agriculteurs. Voir la section «Validité interne et externe ».
}

qui engagent l'exploitation (choix d'investissement par exemple). La mise en place de l'incitation financière peut être limitée par les contraintes budgétaires de l'expérimentateur. En outre, les expériences impliquant des acteurs professionnels sont plus chronophages et peuvent impliquer des obstacles administratifs. Ces complications peuvent pousser à la réalisation d'expériences sans incitations monétaires (Reynaud et Couture, 2012).

Dans le domaine de l'environnement, le bien public et son financement peuvent être représentés par un jeu de biens publics, un jeu de ressources communes ou, plus largement, un jeu comprenant des décisions collectives. Dans le cadre de ces jeux, les incitations monétaires sont faciles à mettre en place. Quand il s'agit de tester des politiques publiques de l'environnement, les expériences couramment réalisées sont des expériences en laboratoire avec des participants qui peuvent être des étudiants (Dubois et al., 2015) ou des agriculteurs (Rozan et al., 2015), selon le public visé par la politique testée. La réalisation de ce type d'expériences sur le terrain est compliquée par l'importance des décisions de groupes qui sont difficilement observables. Notons également que représenter la contribution à l'environnement par un jeu de biens publics peut être réducteur, l'environnement englobant bien des dimensions (interactions entre compartiments de l'environnement, dimension spatiale, temporelle, etc.).

Dans le domaine de l'alimentation, il est principalement question d'attribuer une valeur économique aux caractéristiques des produits agroalimentaires, de comprendre les choix de composition des caddies et l'impact d'une information. Pour mesurer les valeurs, les expériences consistent pour les participants à prendre part à des enchères. Ces enchères sont incitées, c'està-dire occasionnent de véritables ventes, permettent de révéler la vraie valeur que 
chaque consommateur attribue au produit ou à des caractéristiques de produits. Pour observer des comportements d'achats, les chercheurs recourent à des magasins réels ou virtuels en laboratoire pour créer un contexte plus proche des conditions réelles d'achat. Ainsi, les préférences des consommateurs sont révélées non seulement à l'échelle du produit mais aussi à l'échelle du panier. Le magasin expérimental offre un degré de contrôle de l'environnement et des décisions observées plus important que l'étude de terrain. Il permet de considérer des situations contrefactuelles comme l'introduction d'un produit non commercialisé ou un label encore inexistant. Ainsi, il est par exemple possible de tester l'impact de nouveaux formats d'étiquetage (Lacroix et al., 2015) ou l'efficacité de labels écologiques (Bazoche et al., 2015) sur des décisions d'achats réelles, tout en observant le consommateur dans un environnement contrôlé.

Comme la mise en place d'incitations monétaires a tendance à limiter le biais hypothétique, la tentation d'introduire absolument un système d'incitations monétaires est grande, d'autant que la communauté expérimentale en fait un passage obligé pour les publications spécialisées (Roth, 1993). Pourtant, en s'appuyant sur 74 études expérimentales publiées dans des revues d'économie prestigieuses, les conclusions de Camerer et Hogarth (1999) sont moins tranchées quant à leur efficacité.

Si elles permettent d'améliorer la motivation des participants pour réduire les irrationalités économiques (Burke et al., 1996 ; Bohm, 1994 ; Brookshire et Coursey, 1987) et d'estimer plus précisément les préférences réelles des individus (Harrison et al., 2004), elles peuvent se montrer inutiles, voire nuisibles dans certains cas (Etchart-Vincent, 2006). D'une part, il est montré que les écarts comportementaux peuvent être faibles voire négligeables (Slovic, 1969 ; Tversky et Kahneman, 1992). D'autre part, le processus d'incitations peut altérer la perception de la tâche par les participants en capturant excessivement leur attention sur les rémunérations monétaires au détriment de la tâche proposée, même lorsqu'elle est relativement simple. Bardsley et al. (2010) mettent en garde contre une présomption que toutes les tâches expérimentales doivent intégrer des incitations monétaires. Les incitations financières sont presque toujours justifiées lorsque les expériences testent la rationalité des comportements, étudient des comportements avec une dimension de désirabilité sociale forte, ou encore testent des modèles théoriques dont la structure des incitations monétaires est parfaitement en adéquation avec les objectifs de la tâche proposée. Mais il existe des cas, notamment lorsque la recherche est plus appliquée, où il est productif de mettre de côté les conventions pour ne pas créer de nouveaux artefacts qui ne peuvent être contrôlés. En économie agricole, il est parfois nécessaire de faire un arbitrage qui peut conduire à limiter, éventuellement à ne pas utiliser, les incitations monétaires dans les expériences, notamment lorsque les préférences ne peuvent être révélées par le marché (e.g. mesure de disposition à payer pour un produit innovant) ou lorsque le contexte ne peut pas être reproduit en laboratoire (e.g. observation de comportements face au risque d'incendie).

\section{Validité interne et externe}

Un autre défi méthodologique pour l'économie expérimentale concerne les questions de validité interne et de validité externe des travaux expérimentaux. La méthode expérimentale en économie est fondée sur le principe du contrôle, à la fois des variables et de l'environnement. Ce contrôle est central pour étudier l'impact causal d'une mesure sur les comportements individuels et pour avoir une forte 
validité interne. Cette méthode permet en particulier d'analyser des phénomènes économiques pour lesquels il n'y a pas de données ou pour lesquels les données sont difficilement observables ou exploitables. Pour l'expérimentateur, l'expérience en laboratoire permet un degré de contrôle plus élevé mais une capacité de généralisation des résultats (qui permet une forte validité externe) plus faible que l'expérience de terrain.

Pour définir ces notions de validité plus précisément, nous reprenons la présentation synthétique de Falk et Heckman (2009). Supposons qu'un chercheur souhaite déterminer l'effet d'une variable dénotée $X_{1}$ sur une variable dénotée $Y$ sachant qu'il existe une fonction $f$ qui lie les deux variables de la manière suivante : $Y=f\left(X_{1}, X_{2}, \ldots, X_{\mathrm{n}}\right)$. Le chercheur étudie donc l'effet de variations de la variable $X_{1}$ sur la variable $Y$ étant donné un niveau donné des autres variables $\tilde{X}=\left(\tilde{X}_{2}, \ldots \tilde{X}_{n}\right)$. Nous pouvons alors définir la validité interne comme le niveau de contrôle du chercheur sur les variables $\tilde{X}$ quand il détermine la causalité de sorte que c'est bien l'effet de la variable $X_{1}$ sur la variable dénotée $Y$ qu'il mesure. Et nous pouvons définir la validité externe comme le degré de maintien de la causalité entre ces deux variables aussi bien pour un niveau $\tilde{X}$ des autres variables que pour un autre niveau de ces variables, par exemple $\dot{X}=\left(\dot{X}_{2}, \ldots, \dot{X}_{\mathrm{n}}\right)$.

Dans les travaux présentés lors de l'École Chercheurs (voir tableau 1), les auteurs s'emparent de manière différente des notions de validité. Les articles rattachés aux thèmes agriculture et environnement s'appuient tous sur un modèle structurel - c'est-à-dire un modèle théorique validé - qui leur permet de s'assurer d'une bonne identification des causalités. La différence entre les expériences réalisées dans ces articles réside dans l'échantillon des participants en termes de taille et de population, permettant des résultats plus ou moins robustes et généralisables (Rozan et al., 2015). Comme mentionné dans la section précédente, les incitations financières dans l'expérience peuvent être trop faibles, ce qui rend les résultats moins généralisables. De plus, la validité externe peut être mise à mal du fait de biais de sélection. En effet, la constitution d'un échantillon de professionnels (agriculteurs) oblige souvent au recours à des organismes tiers (chambre d'agriculture, coopératives), ce qui pose la question du biais de sélection (Mantilla et al., 2015).

Les travaux sur l'alimentation cherchent à révéler les préférences des consommateurs en rendant exogène l'impact de l'information sur les choix. Ces travaux s'appuient soit sur des choix avec un apport simultané de l'information dans un magasin réel ou virtuel (Bazoche et al., 2015), soit sur des choix avec une révélation progressive et contrôlée de l'information (Lacroix et al., 2015). La question de la généralisation des résultats peut se poser du fait que le cadre expérimental focalise l'attention des sujets sur le phénomène étudié. Une forte validité interne reste néanmoins assurée en contrôlant les variables explicatives et en isolant, ainsi, les effets de causalité. Enfin, un des gros enjeux en alimentation est d'identifier les décisions d'achat par rapport aux décisions de consommation puisque celui qui achète n'est pas forcément celui qui consomme et ce qui est acheté n'est pas forcément consommé.

Objet de l'attention des expérimentateurs depuis toujours, la validité externe a été le sujet d'un vif débat dans la littérature à la suite de la publication de deux articles : Levitt et List (2007a et b). Ces auteurs considèrent la validité externe comme fondamentale et mettent en doute la validité externe des expériences menées en laboratoire avec des étudiants. Leur critique porte sur plusieurs points. (i) Les 
sujets ont conscience qu'ils sont observés : les sujets de l'expérience savent qu'ils sont observés par des chercheurs et sont donc plus susceptibles d'adapter leur comportement en fonction de ce qu'ils pensent être l'objectif de l'expérience. Le chercheur pourrait ainsi mettre en évidence un effet significatif qui ne serait qu'un artefact. L'ajout de contexte, comme dans les études de Bazoche et al. (2015) et de Lacroix et al. (2015), permet de réduire cet artefact. (ii) Le contexte de la décision : il y a de grandes différences entre le contexte auquel sont soumis des étudiants en laboratoire et le contexte dans lequel évoluent les agriculteurs ou les consommateurs. L'ajout de contexte dans l'expérience et l'utilisation de sujets non standards, comme dans les études de Reynaud et Couture (2012), de Mantilla et al. (2015) et de Rozan et al. (2015), permettent de réduire ces effets. (iii) L'autosélection des participants : les étudiants ne participent pas aux expériences de manière aléatoire. Ceux qui participent aux expériences en laboratoire de manière volontaire ont donc des caractéristiques particulières. Cette autosélection peut biaiser les résultats. Ainsi, les études comme celle de Dubois et al. (2015) peuvent comme toutes les expériences utilisant des étudiants en laboratoire souffrir de ce biais. (iv) Le niveau des enjeux monétaires : comme mentionné précédemment, les enjeux monétaires peuvent être très différents des enjeux auxquels font face les agents économiques.

Les économistes agricoles, utilisant ou non l'économie expérimentale, sont familiers de ce type de critiques qui font écho à des réflexions plus générales sur les biais de sélection, le biais hypothétique ou le biais stratégique, ou encore sur le biais de désirabilité sociale dans les enquêtes ou les bases de données existantes. Ces critiques ont donné lieu à des discussions qui peuvent être résumées en quatre points : (i) la généralisation n'est pas un aspect fondamental de l'économie expérimentale. Dans bien des cas, le but est de tester des théories économiques sans nécessité de généralisation à d'autres contextes (Camerer, 2015 ; Kessler et Vesterlund, 2015). Nous retrouvons largement cet objectif théorique dans les travaux de Reynaud et Couture (2012), Mantilla et al. (2015), Rozan et al. (2015) et Dubois et al. (2015). (ii) Si l'objectif est la généralisation, le protocole doit être conçu en ce sens. Il s'agira d'identifier a priori les variables clés qui sont susceptibles de différer entre le laboratoire et le domaine naturel (niveau des enjeux monétaires, degré de communication entre les agents, etc.). C'est aussi cet objectif que visent les auteurs de la majorité des travaux présentés lors de l'École Chercheurs (voir tableau 1), en sélectionnant un échantillon d'agriculteurs ou de consommateurs ou en concevant leur protocole expérimental de manière à prendre en compte l'ensemble des variables qui entrent en jeu dans le mécanisme étudié. (iii) Le problème de la généralisation des résultats n'est pas spécifique à l'économie expérimentale. La notion de généralisation est toujours relative à un contexte précis et aucune étude expérimentale ne peut se vanter d'être parfaitement généralisable à tout contexte naturel (Falk et Heckman, 2009). (iv) Les résultats expérimentaux sont d'abord qualitatifs, et généralisables à d'autres contextes dans ce sens, plutôt que quantitatifs. Kessler et Vesterlund (2015) notent que peu d'expérimentateurs soutiendraient que les résultats expérimentaux sont toujours généralisables quantitativement. Cependant, sur le plan qualitatif, les expériences mettent en évidence les mécanismes en jeu ou révèlent l'influence de variables clés parfois non considérées dans les modèles théoriques. Les travaux en économie agricole font face aux mêmes critiques que dans les autres domaines de l'économie. La question de recherche et les choix méthodologiques déterminent les degrés de validité interne et de validité externe. 


\section{Usage de la tromperie}

Les expérimentateurs ont depuis longtemps préconisé de ne pas utiliser la tromperie (deception en anglais) dans leurs protocoles. Ce choix méthodologique distingue les expériences en économie de celles en psychologie. Ainsi, dans les expériences en économie agricole (et donc dans les travaux présentés dans le tableau 1), la tromperie est bannie. Toutefois, cette règle bien ancrée et peu discutée jusqu'à présent commence à être débattue de sorte que, dans certains cas, la profession admet la possibilité d'utiliser la tromperie dans un protocole expérimental sous certaines conditions.

Pourquoi l'économie expérimentale at-elle toujours insisté sur l'interdiction de tromper les sujets ? Cooper (2014) indique que les raisons sont de deux ordres. Elles concernent, d'une part, le contrôle des incitations dans le cadre de l'expérience menée et, d'autre part, les externalités négatives pour la profession. L'interdiction de l'usage de la tromperie vient essentiellement du postulat que font les économistes que les sujets maximisent une fonction « objectif » lorsqu'ils prennent leurs décisions. De là sont formulées des hypothèses testées en laboratoire. Ces hypothèses ne sont valides que si les sujets maximisent la fonction «objectif» considérée. Si les sujets pensent qu'ils seront rémunérés en fonction de critères autres que ceux qui leur sont donnés dans les instructions, l'expérimentateur perd le contrôle des incitations et donc les hypothèses formulées ne sont plus valides. Afin d'éviter cette perte de contrôle, les expérimentateurs souhaitent éviter la tromperie dans le cadre de l'expérience. Par ailleurs, la profession craint que si une partie des chercheurs utilise la tromperie, l'ensemble de la profession subisse des externalités négatives dans la mesure où les sujets seraient susceptibles de douter des incitations présentées dans toute expérience, même si en réalité elle n'utilise pas la tromperie.
L'article de Cooper (2014) est intéressant à un autre titre. David Cooper est un des éditeurs de la revue du champ, Experimental Economics. Il insiste pour que la communauté des expérimentateurs continue de suivre la recommandation de ne pas utiliser la tromperie et l'exige pour toute soumission à la revue de la discipline. Cependant, il reconnaît ${ }^{7}$ : «Cela dit, seul un extrémiste soutiendrait que les expérimentalistes (ou les économistes en général) ne devraient jamais utiliser la tromperie » (Cooper, 2014). Il mentionne quatre raisons pour lesquelles l'usage de la tromperie pourrait être toléré dans des travaux thématiques : (i) la tromperie ne doit pas léser le sujet au-delà de ce qui existe dans une expérience sans tromperie; (ii) les auteurs doivent démontrer que le coût d'une expérience sans tromperie est prohibitif ; (iii) les expérimentateurs doivent organiser un débriefing après l'expérience pour expliquer l'usage nécessaire de la tromperie; et (iv) les résultats attendus ont une valeur telle que l'expérience doit être menée même au prix de l'usage de la tromperie.

La position de David Cooper de ne pas accepter de travaux expérimentaux utilisant la tromperie pour publication dans Experimental Economics est aussi la position de revues de premier rang telles que l'American Economic Review et le Journal of Economic Behavior and Organization. L'American Journal of Agricultural Economics (AJAE) a également adopté cette position en 2011. Cependant, elle a récemment assoupli ce critère (Colson et al., 2016; Krawczyk, 2013 ; Rousu et al., 2015). Afin d'éclairer le comité éditorial et les auteurs susceptibles de soumettre à l'AJAE, Colson et al. (2016) ont réalisé une enquête auprès de chercheurs expérimentateurs et d'étudiants pour déterminer l'acceptabilité, au sein de ces deux

7. Traduction des auteurs. 
populations, de l'usage de la tromperie. Les résultats indiquent que tromper les sujets avec pour conséquence possible un traumatisme, ne pas payer les sujets alors qu'une rémunération leur avait été promise ou proposer des produits avec un label trompeur sont considérées comme des pratiques inacceptables, mais que tromper les sujets sur l'objectif de l'étude, observer les participants à leur insu, donner une information incomplète sur un produit vendu dans une enchère expérimentale et mentir sur le fait que certains sujets dans l'expérience ne sont pas des sujets naïfs mais sont contrôlés par l'expérimentateur sont considérées comme des pratiques acceptables. Les résultats de Colson et al. (2016) sont en adéquation avec les résultats de Krawczyk (2013) et de Rousu et al. (2015) qui s'accordent sur le fait que les pratiques qui utilisent la tromperie par omission sont plus acceptables que celles qui l'utilisent de manière explicite.

\section{L'économie expérimentale comme outil d'aide à la décision}

Cette section s'intéresse à la manière dont les travaux académiques en économie agricole utilisant l'économie expérimentale peuvent être utiles à des organismes publics ou privés dans leur conception de politiques incitatives. Lors de l'École Chercheurs, deux économistes expérimentaux ont discuté la façon dont l'économie expérimentale peut contribuer à la prise de décision en matière de politiques publique (Lefebvre, 2015) ou privée (Saulais, 2015).

\section{Outil d'aide à la décision pour les pouvoirs publics}

Lefebvre (2015) rapporte, à partir de son expérience à la Direction générale de l'Agriculture et du Développement Rural (DG AGRI) à la Commission européenne, que pendant longtemps les méthodes expérimentales ont été mal appréciées dans l'évaluation économique des politiques agricoles. Ce n'est que récemment qu'une série de documents de travail ainsi qu'un workshop organisé au sein de la DG AGRI ont contribué à une prise de conscience de l'intérêt de ces méthodes (van Bavel et al., 2013, 2015 ; Colen et al., 2016). Les discussions ont ainsi aidé à identifier les différentes méthodes expérimentales utilisées en économie et ont permis de souligner leurs bénéfices, limites et complémentarités comparées aux méthodes conventionnelles.

Marianne Lefebvre souligne que le critère principal de la DG AGRI, quant à une nouvelle méthode scientifique, est son utilité pour l'évaluation de politiques. Les besoins principaux d'évaluation concernent l'examen ex ante et l'observation ex post de politiques, la comparaison des effets des changements incrémentaux des politiques présentes avec l'introduction de nouvelles mesures, la prise en compte de l'hétérogénéité dans les politiques en vigueur dans l'ensemble de l'UE ainsi que la participation des parties concernées. Les demandes récentes, plus spécifiques, de la DG AGRI pour améliorer les évaluations concernent notamment des analyses au niveau des exploitations agricoles, la prise en compte de la nature volontaire et du caractère purement réglementaire (sans participation de l'UE) de beaucoup de mesures ainsi que le respect des spécificités des États membres dans la mise en œuvre de la politique agricole commune. Tandis que les méthodes conventionnelles sont basées sur l'analyse de données officielles représentatives ou de marché, l'intérêt des expériences est de directement collecter des données liées à la question ciblée. À l'occasion du workshop mentionné précédemment, les membres de la DG AGRI ont manifesté leur intérêt prioritaire pour les tests ex ante de nouvelles politiques, bien avant l'analyse du comportement des agriculteurs vis-à-vis des politiques et la mesure de l'impact net, ex post donc, d'une politique particulière. 
Marianne Lefebvre compare ensuite quatre méthodes évoquant leurs bénéfices, limites et complémentarités à partir d'exemples de travaux. Il s'agit des expériences de choix, en laboratoire, de terrain ${ }^{8}$ et des essais randomisés contrôlés (randomized controlled trial [RCT]). Une expérience de choix consiste en une enquête sur la valorisation d'une mesure ou d'un bien hypothétique, par exemple un nouveau produit ou une nouvelle technologie ou politique. Kuhfuss et al. (2012) montrent, à partir de cette méthode, l'importance des normes sociales influençant l'adoption d'une mesure agro-environnementale par des viticulteurs. Utilisant la même méthode, Schulz et al. (2014) étudient l'acceptabilité de mesures de verdissement de la politique agricole commune et trouvent que les agriculteurs avec les terres les plus productives et les productions les plus intenses ont le moins tendance à accepter les mesures les plus contraignantes. Les expériences en laboratoire sont rémunérées en fonction des choix des participants et les paiements reçus sont donc différents. Les expériences sont présentées hors contexte et exécutées avec des étudiants. Dans ce cadre, Le Coent et al. (2014) se focalisent sur l'impact de l'adoption et la performance environnementale de plusieurs modes de paiement de subventions conditionnelles, telles que des mesures agro-environnementales, en examinant l'importance des croyances sur les contributions des autres participants, l'aversion individuelle pour le risque et la réciprocité.

Contrairement à ces deux premiers types d'expérience, les expériences de terrain n'ont pas encore été utilisées en lien direct avec des aspects de la Politique agricole commune (PAC) (uniquement pour

8. Il ne s'agit pas ici des expériences de terrain au sens général mais de la traduction française du terme field experiments, c'est-à-dire les types (ii) à (iv) présentés dans l'introduction et basés sur la classification de Harrison et List (2004). la mesure de préférences pour le risque et l'incertitude, Bocquého et al. [2014] ; Bougherara et al. [2017]) et les RCT se concentrent essentiellement sur les pays en développement. Un RCT compare les choix ou comportements d'individus qui ont été aléatoirement affectés à un groupe soumis à un traitement sous forme d'une politique ou d'un programme particulier aux choix ou comportements d'un groupe comparable qui n'est pas soumis à ce traitement. À partir de cette méthode, Duflo et al. (2011) trouvent que l'efficacité relative de différentes politiques pour stimuler l'usage d'engrais tout en limitant son usage excessif par des agriculteurs kenyans dépend particulièrement de la cohérence de leurs choix inter-temporels et de leur impatience.

En conclusion du workshop mentionné, Marianne Lefebvre souligne que l'avantage de l'usage de méthodes expérimentales dans l'évaluation de politiques publiques pourrait particulièrement se trouver dans la complémentarité avec les méthodes plus conventionnelles d'analyse de données observées. La validité externe étant centrale pour les pouvoirs publics, les expériences économiques pourraient être abordées à partir d'une approche incrémentielle qui commencerait avec des tests de protocoles en laboratoire pour procéder ensuite à des expériences de terrain. Les méthodes expérimentales sont aussi particulièrement adaptées à l'évaluation ex ante de mesures de politiques publiques. Pour l'évaluation ex post, la panoplie de méthodes, déjà riche, que constituent les analyses économétriques de données observées et de réponses à des enquêtes pourrait s'enrichir de RCT. Le niveau de difficulté pour collecter les données est comparable entre les méthodes économétriques d'analyse de données observées et les méthodes expérimentales. Cependant, l'interprétation des résultats est plus facile si les données sont collectées à l'aide d'expériences, puisqu'elles permettent plus de contrôle. 


\section{Outil d'aide à la décision en entreprise}

Saulais (2015) travaille depuis six ans au centre de recherche de l'Institut Paul Bocuse. Les recherches de cet institut sont centrées sur la relation homme-aliment et caractérisées par une approche pluridisciplinaire appliquée en situation réelle, en restaurant expérimental par exemple. Les grands thèmes de recherche sont la restauration durable, la créativité culinaire et la symbolique du repas. Dans le cadre de ses trois missions de recherche, de formation et d'exécution d'études appliquées, l'institut bénéficie de nombreux contacts avec d'autres entreprises autour de ses thématiques.

L'économie expérimentale est mobilisée dans ce cadre selon trois types principaux de demande: l'évaluation d'un produit ou service, l'évaluation du mode de présentation d'une offre et l'impact du contexte. Les travaux permettent de suggérer des éclairages stratégiques par rapport au fonctionnement d'une filière, d'un marché ou d'une organisation. L'évaluation d'un produit ou service consiste à mesurer les éléments sources de valeur dans la conception d'un produit ou d'un service. Il s'agit de connaître et de prédire les préférences des consommateurs afin de proposer une offre adaptée. Un accent particulier est mis sur la validité externe des résultats en proposant de véritables produits et en fournissant les informations réelles relatives à sa conception. En termes d'approche expérimentale, la disposition à payer pour le produit ou service en question est révélée à partir de choix incités monétairement, souvent en complément d'une évaluation sensorielle. Les études sur le mode de présentation et l'impact du contexte visent à améliorer la conception d'offres, de menus ou de gammes. L'approche expérimentale mesure également les dispositions à payer et insiste sur les aspects comportementaux en tenant compte du contexte économique ou institutionnel d'une offre culinaire (par exemple position de l'aliment dans le menu, type de menu proposé par défaut). Dans ce cadre, les expériences économiques avec des collaborateurs ou différents maillons de la filière complètent des études d'économie industrielle ou des organisations afin de donner des éclairages stratégiques sur l'intérêt d'étendre une gamme de produits par exemple.

Laure Saulais illustre les études menées sur le mode de présentation à partir d'une expérience réalisée dans le cadre d'une thèse CIFRE (Convention industrielle de formation par la recherche) (Jegou, 2014). En lien avec le café, produit à forte valeur ajoutée, l'étude s'est intéressée à l'étendue optimale de l'offre de variétés et au type de gamme à proposer (origines ou préparations). La question de l'étendue optimale a été étudiée à partir d'un questionnaire sur la connaissance, l'utilisation et l'intérêt pour le café avec 161 participants dans un restaurant expérimental et sur un campus universitaire. L'étude permet de montrer que l'étendue souhaitable (proposition de choix entre trois cafés ou entre huit cafés) dépend du genre des participants et de leur proximité à la catégorie des produits. Par exemple, alors que la majorité des femmes préfère le choix entre trois types de cafés, la majorité des hommes préfère choisir entre huit types de cafés. La question relative au type de gamme a été étudiée à partir de la disposition à payer avec incitations monétaires pour un café choisi dans le menu, pioché au hasard dans la carte ou proposé par le barista. Selon le traitement, l'origine ou le type de préparation était indiqué. Au total, 231 clients du restaurant expérimental ont été confrontés à ces traitements durant trois semaines consécutives. Les résultats montrent que la disposition à payer est supérieure lorsque le choix est proposé par le barista et lorsque l'origine est indiquée.

Laure Saulais résume que l'économie expérimentale peut servir aux entreprises, 
d'une part comme méthode pour obtenir des résultats appliqués et, d'autre part, de manière exploratoire, pour élargir la réflexion stratégique et fournir de nouvelles perspectives (Saulais et al., 2017). Pour Laure Saulais, les expériences économiques dans ce contexte sont en amont des études marketing et ne visent pas à prédire les prix de vente. Bien que la contribution potentielle directe aux décisions d'entreprises puisse avoir un attrait particulier pour un chercheur, la valorisation scientifique peut être confrontée à des problèmes liés à la pluridisciplinarité de ces recherches, à la confidentialité des résultats et à l'intérêt scientifique parfois limité de contributions destinées à des problèmes spécifiques aux défis des entreprises.

$*$
$* \quad *$

L'École Chercheurs organisée par le département SAE2 de l'INRA avait pour objectif d'apporter une meilleure vision de l'utilisation de l'économie expérimentale afin de traiter des problématiques relatives à l'économie agricole. Les travaux présentés donnent une idée du type et de la variété des questions de recherche auxquelles cette méthodologie est susceptible de répondre avec rigueur scientifique. Ils ont également permis de mettre en évidence les questions méthodologiques sous-jacentes à ce type de recherches. À l'INRA, et plus généralement en économie agricole, les questions des incitations monétaires, de la validité interne et externe et de l'usage de la tromperie se posent particulièrement du fait du caractère finalisé des recherches menées. Des arbitrages peuvent être nécessaires. Dans ce cas, ils doivent être explicites et faire l'objet d'une discussion de la nature du biais introduit et de son impact sur l'interprétation des résultats. Les interventions concernant l'économie expérimentale comme outil d'aide à la décision exposent les attentes d'organismes publics et privés et discutent des arbitrages quant à l'implication des chercheurs (difficultés de publication du fait du caractère peu académique, de la confidentialité des résultats, etc.).

Il n'est pas possible de rendre compte de l'ensemble des présentations et discussions de l'École Chercheurs. Toutefois, notons qu'un théoricien (Treich, 2015) et un économètre (Carpentier, 2015) ont présenté leur vision de l'économie expérimentale. En effet, l'économétrie à partir de bases de données publiques ou privées est depuis toujours la première source de résultats empiriques économiques à l'INRA et plus généralement en économie agricole. Or des complémentarités pourraient apparaître. En effet, la méthode expérimentale apporte de nouvelles données qui viennent en complément des données observées et permettent d'apporter des réponses aux questions de recherche jusque-là encore peu abordées. De plus, les approches économétriques mobilisées pour identifier l'effet causal des variables ciblées sont souvent très complexes, notamment du fait du manque de contrôle des données observées. Ainsi, l'économétrie structurelle très largement utilisée en économie agricole est souvent critiquée pour son grand nombre d'hypothèses, pour les problèmes de variations propres aux facteurs de mesure des effets et pour la non-prise en compte fréquente de l'hétérogénéité des individus (Angrist et Pischke, 2010). Dans ce contexte, l'économie expérimentale pourrait permettre d'étudier plus en détail tant des aspects causaux que méthodologiques.

Bien qu'il existe de nombreux défis à la fois méthodologiques et pratiques à relever, les perspectives de recherche en économie expérimentale appliquée à l'économie agricole sont vastes. En conclusion, nous notons deux pistes qui nous semblent particulièrement prometteuses. D'une part, accentuer le recours à l'économie 
RECHERCHE

Douadia BOUGHERARA, Marielle BRUNETTE, Christoph HEINZEL, Lisette IBANEZ,

Laurent MULLER, Sabrina TEYSSIER

expérimentale comme outil d'aide à la décision s'avère être une piste de recherche à privilégier. De nombreuses recherches en économie expérimentale appliquées à des questions agricoles ont déjà mené à des recommandations précises en termes de politiques publiques. De tels travaux ont été publiés dans de bonnes revues internationales. Par exemple, des recommandations ont été faites concernant les incitations favorisant l'organisation spatiale des terres agricoles (Parkhurst et Shogren, 2007), les mécanismes d'allocation initiale des droits à polluer (Goeree et al., 2010), ou encore les conséquences des taxes alimentaires (Muller et al., 2017). L'enjeu dans ce contexte étant la validité des implications politiques des travaux, la question de la représentativité des échantillons, mobilisant des agriculteurs ou des consommateurs, et donc la question de l'accès à ces populations mérite une attention particulière. De manière générale, afin d'obtenir plus de précisions dans les résultats, la taille des échantillons devrait être élargie.

D'autre part, croiser l'économie expérimentale avec d'autres disciplines en sciences humaines et sociales, comme la psychologie, la sociologie ou la neurologie, mais aussi son association avec l'agronomie ou les sciences de l'ingénieur offrent des perspectives de recherche intéressantes. Par exemple, le croisement avec la psychologie et la neurologie peut enrichir l'étude de la formation des préférences individuelles et collectives; l'analyse du respect de certaines normes ou de règles sociales fait écho aux travaux de sociologues ; enfin, le croisement avec l'agronomie permettrait de tester l'acceptabilité de nouveaux produits tels que les OGM ou de nouvelles variétés plus adaptées au changement climatique, etc. Ce type d'études pourrait déboucher sur des recommandations concernant les enjeux majeurs des années à venir comme le changement climatique, le développement durable...

Ainsi, il semble que la méthodologie expérimentale en économie agricole ne peut être contournée aujourd'hui et doit au contraire être favorisée afin de compléter la boîte à outils disponible pour les économistes agricoles.

Les discussions et analyses de cet article sont issues de l'École Chercheurs "Économie Expérimentale : État des Lieux et Dynamiques de Recherche à I'INRA " organisée par les auteurs à la demande du département SAE2 de I'INRA à Montpellier du 30 septembre au 2 octobre 2015. Elles n'engagent que les auteurs. Nous remercions tous les intervenants ainsi que les participants à cette École. Nous remercions également Sylviane Fortun, Patricia Manzano et Françoise Werdenberg de la Formation Permanente Nationale de I'INRA pour leur soutien dans I'organisation de l'École.

\section{RÉFÉRENCES BIBLIOGRAPHIQUES}

Angrist J.D., Pischke J. (2010). The Credibility Evolution in Empirical Economics: How better Research Design is Taking the Con out of Econometrics. Journal of Economic Perspectives, vol. 24, $\mathrm{n}^{\circ}$ 2, pp. 3-30.

Bardsley N., Cubitt R., Loomes G., Moffatt P., Starmer C., Sugden R. (2010). Experimental Economics: Rethinking the Rules. Princeton University Press.
Bazoche P., Issanchou S., Maratray J., Ginon E. (2015). Evaluating Consumers' Sustainable Choice of Wine: An Online Shop Experiment. Présentation lors de l'École Chercheurs INRA Économie Expérimentale, 30 septembre-2 octobre, Montpellier, France.

Bocquého G., Jacquet F., Reynaud A. (2014). Expected Utility or Prospect 
Theory Maximisers? Assessing Farmers' Risk Behaviour from Field-Experiment Data, European Review of Agricultural Economics, vol. 41, $\mathrm{n}^{\circ}$ 1, pp. 135-172.

Bohm P. (1994). Time Preference and Preference Reversal among Experienced Subjects: The Effects of Real Payments. The Economic Journal, vol. 104, pp. 1370-1378.

Bougherara D., Gassmann X., Piet L., Reynaud A. (2017). Structural Estimation of Farmers' Risk and Ambiguity Preferences: An Field Experiment, European Review of Agricultural Economics, vol. 44, $\mathrm{n}^{\circ} 5$, pp. 782-808.

Brookshire D.S., $\quad$ Coursey D.L. (1987). Measuring the Value of a Public Good: An Empirical Comparison of Elicitation Procedures. The American Economic Review, vol. 77, $\mathrm{n}^{\circ}$ 4, pp. 554-566.

Burke M.S., Carter J.R., Gominiak R.D., Ohl D.F. (1996). An Experimental Note on the Allais Paradox and Monetary Incentives. Empirical Economics, vol. 21, $\mathrm{n}^{\circ} 4$, pp. 617-632.

Camerer C.F. (2015). The Promise and Success of Lab Field Generalizability in Experimental Economics: A Critical Reply to Levitt and List. In Handbook of Experimental Economic Methodology, Oxford University Press.

Camerer C.F., Fehr E. (2004). Measuring Social Norms and Preferences using Experimental Games: A Guide for Social Scientists. Foundations of Human Sociality: Economic Experiments and Ethnographic Evidence from Fifteen Small-Scale Societies, vol. 97, pp. 55-95.

Camerer C.F., Hogarth R.M. (1999). The Effects of Financial Incentives in Experiments: A Review and Capital-LaborProduction Framework. Journal of Risk and Uncertainty, vol. 19, $\mathrm{n}^{\circ}$ 1-3, pp. 7-42.

Carpentier A. (2015). Quelques réflexions d'un micro-économètre appliqué. Présentation lors de l'École Chercheurs INRA Économie Expérimentale, 30 septembre-2 octobre, Montpellier, France.

Colen L., Gomez y Paloma S., LataczLohmann U., Lefebvre M., Préget R., Thoyer S. (2016). Economic Experiments as a Tool for Agricultural Policy Evaluation: Insights from the European CAP. Canadian Journal of Agricultural Economics, vol. 64, $\mathrm{n}^{\circ}$ 4, pp. 667-694.

Colson G., Corrigan J.R., Grebitus C., Loureiro M.L., Rousu M.C. (2016). Which Deceptive Practices, if any, should be allowed in Experimental Economics Research? Results from Surveys of Applied Experimental Economists and Students. American Journal of Agricultural Economics, vol. 98, n² 2, pp. 610-621.

Cooper D.J. (2014). A Note on Deception in Economic Experiments. Journal of Wine Economics, vol. 9, $\mathrm{n}^{\circ}$ 2, pp. 111-114.

Dubois D., Farolfi S., Tidball M., Desolé M., Hofstetter A. (2015). Does Environmental Connotation Affect Coordination Issues in an Experimental Stag Hunt Game? Présentation lors de l'École Chercheurs INRA Économie Expérimentale, 30 septembre-2 octobre, Montpellier, France.

Duflo E., Kremer M., Robinson J. (2011). Nudging Farmers to use Fertilizer: Theory and Experimental Evidence from Kenya. The American Economic Review, vol. 101, $\mathrm{n}^{\circ}$ 6, pp. 2350-2390.

Etchart-Vincent N. (2006). Expériences de laboratoire en économie et incitations monétaires, Revue d'économie politique, vol. 116, $n^{\circ} 3$, pp. 383-418.

Falk A., Heckman J. (2009). Lab Experiments are a Major Source of Knowledge in the Social Sciences, Science, vol. 326, $\mathrm{n}^{\circ} 5952$, pp. 535-538.

Frappier L. (2015). Analyse bibliométrique des publications en économie expérimentale. Présentation lors de l'École Chercheurs INRA Économie Expérimentale, 30 septembre-2 octobre, Montpellier, France.

Goeree J.K., Palmer K., Holt C.A., Shobe W., Burtraw D. (2010). An Experimental Study of Auctions versus Grandfathering to Assign Pollution Permits. Journal of the European Economic Association, vol. 8, $\mathrm{n}^{\circ} 2-3$, pp. 514-525.

Hamermesh D.S. (2013). Six Decades of Top Economics Publishing: Who and How? Journal of Economic Literature, vol. 51, $\mathrm{n}^{\circ} 1$, pp. 162-172. 
RECHERCHE

Douadia BOUGHERARA, Marielle BRUNETTE, Christoph HEINZEL, Lisette IBANEZ,

Laurent MULLER, Sabrina TEYSSIER

Harrison G.W., $\quad$ List J.A. (2004). Field Experiments. Journal of Economic Literature, vol. 42, n 4, pp. 1009-1055.

Harrison G.W., Harstad R.M., Rutström E.E. (2004). Experimental Methods and Elicitation of Values. Experimental Economics, vol. 7, n 2, pp. 123-140.

Holt C.A., Laury S.K. (2002). Risk Aversion and Incentive Effects. The American Economic Review, vol. 92, n 5 , pp. 16441655.

Jegou C. (2014). Perception et valorisation $d u$ café dans la restauration: comment concevoir et mettre à disposition une offre à forte valeur ajoutée ? Thèse, Université de Grenoble, 2014.

Kagel J.H., Roth A.E. (1995). The Handbook of Experimental Economics. Princeton, Princeton University Press.

Kessler J.B., Vesterlund L. (2015). The External Validity of Laboratory Experiments: The Misleading Emphasis on Quantitative Effects. In Handbook of Experimental Economic Methodology. Oxford University Press.

Krawczyk M. (2013). Delineating Deception in Experimental Economic: Researchers' and Subjects' Views. Faculty of Economic Sciences Working Paper, University of Warsaw.

Kuhfuss L., Jacquet F., Préget R., Thoyer S. (2012). Le dispositif des MAEt pour l'enjeu eau: Une fausse bonne idée? Revue d'études en agriculture et environnement, vol. $93, \mathrm{n}^{\circ} 4$, pp. 395-422.

Lacroix A., Muller L., Ruffieux B. (2015). Environmental Labelling and Consumption Changes: A Food Choice Experiment. Présentation lors de l'École Chercheurs INRA Économie Expérimentale, $30 \mathrm{sep}$ tembre-2 octobre, Montpellier, France.

Le Coent P., Préget R., Thoyer S. (2014). Why Pay for Nothing? An Experiment on a Conditional Subsidy Scheme in a Threshold Public Good Game. Economics Bulletin, vol. 34, n 3, pp. 1976-1989.

Lefebvre M. (2015). L'économie expérimentale comme aide à la décision dans les politiques publiques. Exemple de la Politique agricole commune. Présentation lors de l'École Chercheurs INRA Économie Expérimentale, 30 septembre- 2 octobre, Montpellier, France.

Levitt S.D， List J.A. (2007a). Viewpoint: On the Generalizability of Lab Behaviour to the field. Canadian Journal of Economics, vol. $40, \mathrm{n}^{\circ}$ 2, pp. 347-370.

Levitt S.D, List J.A. (2007b). What do Laboratory Experiments Measuring Social Preferences Reveal about the Real World? Journal of Economic Perspectives, vol. 21, $\mathrm{n}^{\circ} 2$, pp. 153-174.

Loomis J. (2011). What's to Know about Hypothetical Bias in Stated Preference Valuation Studies? Journal of Economic Surveys, vol. 25, $\mathrm{n}^{\circ}$ 2, pp. 363-370.

Lusk J.L., McLaughlin L., Jaeger S.R. (2007). Strategy and Response to Purchase Intention Questions. Marketing Letters, vol. 1-2, $\mathrm{n}^{\circ} 18$, pp. 31-44.

Mantilla C., Hopfensitz A., Miquel-Florensa J. (2015). Conditional Contracts in Open access Fisheries: Can they Solve the Commons Problem? Présentation lors de l'École Chercheurs INRA Économie Expérimentale, 30 septembre-2 octobre, Montpellier, France.

Mitani Y., Flores N.E. (2014). Hypothetical Bias Reconsidered: Payment and Provision Uncertainties in a Threshold Provision Mechanism. Environmental and Resource Economics, vol. 59, $\mathrm{n}^{\circ} 3$, pp. 433-454.

Muller L., Lacroix A., Lusk J.L., Ruffieux B. (2017). Distributional Impacts of Fat Taxes and Thin Subsidies. Economic Journal, vol. 127, pp. 2066-2092.

Ostrom, E. (2009). The Prize in Economic Sciences 2009 - Advanced Information. Nobelprize.org. Nobel Media AB 2014. Web. 6 Nov 2017. <http://www.nobelprize. org/nobel_prizes/economic-sciences/laureates/2009/advanced.html>

Parkhurst G.M., Shogren J.F. (2007). Spatial Incentives to Coordinate Contiguous Habitat. Ecological Economics, vol. 64, $\mathrm{n}^{\circ} 2$, pp. 344-355.

Plott C.R. (1982). Industrial Organization Theory and Experimental Economics. Journal of Economic Literature, vol. 20, $\mathrm{n}^{\circ} 4$, pp. 1485-1527. 
Reynaud A., Couture S. (2012). Stability of Risk Preference Measures: Results from a Field Experiment on French Farmers. Theory and Decision, vol. 73, $\mathrm{n}^{\circ} 2$, pp. 203-221.

Roth A.E. (2012). The Prize in Economic Sciences 2012. Nobelprize.org. Nobel Media AB 2014. Web. 6 Nov 2017. <http:// www.nobelprize.org/nobel_prizes/economic-sciences/laureates/2012/>

Roth A.E. (1988). Laboratory Experimentation in Economics: A Methodological Overview. Economic Journal, vol. 98, $\mathrm{n}^{\circ}$ 393, pp. 974-1031.

Roth A.E. (1993). The Early History of Experimental Economics. Journal of the History of Economic Thought, vol. 15, $\mathrm{n}^{\circ} 2$, pp. 184-209.

Rousu M.C., Colson G., Corrigan J.R., Grebitus C., Loureiro M.L. (2015). Deception in Experiments: Towards Guidelines on Use in Applied Economics Research. Applied Economic Perspectives and Policy, vol. 37, $\mathrm{n}^{\circ}$ 3, pp. 524-536.

Rozan A., Bchir A., Cochard F., Sarr H. (2015). Pollution diffuse et taxe ambiante plafonnée. Présentation lors de l'École Chercheurs INRA Économie Expérimentale, 30 septembre-2 octobre, Montpellier, France.

Samuelson P.A., Nordhaus W.D. (1985). Economics. New York: McGraw-Hill, 12th Edition.

Saulais L. (2015). L'économie expérimentale comme outil d'aide à la décision en entreprise. Présentation lors de l'École Chercheurs INRA Économie Expérimentale,
30 septembre-2 octobre, Montpellier, France.

Saulais L., Muller L., Lesgards V. (2017). Murmurer à l'oreille... de l'industriel ? L'économie expérimentale comme outil d'aide à la décision en entreprise. Revue économique, vol. 68 , ${ }^{\circ}$ 5, pp. 147-162.

Schulz N., Gunnar B., Latacz-Lohmann U. (2014). Assessing Farmers' Willingness to Accept Greening: Insights from a Discrete Choice Experiment in Germany. Journal of Agricultural Economics, vol. 65, $\mathrm{n}^{\circ} 1$, pp. 26-48.

Slovic P. (1969). Differential Effects of Real versus Hypothetical Payoffs on Choices among Gambles. Journal of Experimental Psychology, vol. 80, n 3, pp. 434-437.

Treich N. (2015). Some Remarks from $a$ Theorist. Présentation lors de l'École Chercheurs INRA Économie Expérimentale, 30 septembre-2 octobre, Montpellier, France.

Tversky A., Kahneman D. (1992). Advances in Prospect Theory - Cumulative Representation of Uncertainty. Journal of Risk and Uncertainty, vol. 5, $\mathrm{n}^{\circ} 4$, pp. 297-323.

Van Bavel R., Herrmann B., Esposito G., Proestakis A. (2013). Applying Behavioural Sciences to EU Policy-Making. JRC Science and Policy Report.

Van Bavel R., Rodríguez-Priego N., Maghiros I. (2015). Seven Points to Remember when Conducting Behavioural Studies in Support of EU Policy-Making. JRC Science and Policy Report. 